\title{
Erratum to: Strong Gravitational Lensing in a Brane-World Black Hole
}

\author{
GuoPing $\mathrm{Li}^{1} \cdot$ Biao $\mathrm{Cao}^{2} \cdot$ Zhongwen Feng $^{1} \cdot$ \\ Xiaotao $\mathbf{Z u}^{1}$
}

Published online: 5 August 2015

(C) Springer Science+Business Media New York 2015

\section{Erratum to: Int J Theor Phys \\ DOI 10.1007/s10773-015-2545-y}

In this erratum to our published paper, we correct and clarify some of statements. A few equations given in our paper were in fact obtained and published many years ago by Virbhadra's research group. We clarify that those equations were neither obtained by us nor by other authors cited in our paper.

(A) See a paragraph above Eq. 7. There is an equations in this paragraph which is not numbered. Here, we correct our statement and give reference. According to the geometric principle, the relationship between the image and the source can be described by the seminal Virbhadra-Ellis lens equation [1], which is

$$
\tan \theta-\tan \beta=D_{L S} /[\tan \theta+(\alpha-\alpha)] \times D_{O S}
$$

There are many gravitational lens equations, but this by Virbhadra and Ellis is most used because of its accuracy.

(B) The Eq. 7 in our paper was obtained by Virbhadra et al. [2] and is also discussed in [3]. This equation is for light deflection angle in a general space-time (static and spherically symmetric).

The online version of the original article can be found at http://dx.doi.org/10.1007/s10773-015-2545-y.

\section{Biao Cao}

caobiao0301@163.com

GuoPing Li

li_gp2009@163.com

1 School of Physical Electronics, University of Electronic Science and Technology of China, Chengdu, Sichuan 610054, China

2 Institute of Theoretical Physics, China West Normal University, Nanchong, Sichuan 637009, China 


$$
\alpha\left(r_{0}\right)=I\left(r_{0}\right)-\pi, I\left(r_{0}\right)=\int_{r_{0}}^{\infty} \frac{2 \sqrt{B}}{\sqrt{C} \sqrt{\frac{C}{C_{0}} \frac{A_{0}}{A}-1}} d r
$$

We also used the equation for impact parameter of light that was obtained by Virbhadra et al. [2]:

$$
u=r_{0} \sqrt{C\left(r_{0}\right) / A\left(r_{0}\right)}
$$

(C) See the photon sphere equation given in the paragraph below Eq. 7 in our published paper. The equation is not numbered in our paper. This equation was obtained by Virbhadra and Ellis [4] and Claudel, Virbhadra, and Ellis [5] with the help of 2 alternative definitions of photon spheres, see below:

$$
C^{\prime}(r) / C(r)=A^{\prime}(r) / A(r)
$$

Equations discussed above played very significant role when we computed the strong gravitational lensing effects for Brane-World black holes. We also used a term relativistic images and ignored that this was coined by Virbhadra and Ellis [1] and relativistic images were comprehensively studied by Virbhadra [6]. In view of this, we clarify that it is truly important for us to state an erratum, because we ignored stating the provenance of those equations in the published paper.

We are grateful to Dr. K. S. Virbhadra for illuminating correspondence.

\section{References}

1. Virbhadra, K.S., Ellis, G.F.R.: Phys. Rev. D 62, 084003 (2000)

2. Virbhadra, K.S., Narasimha, D., Chitre, S.M.: Astron. Astrophys. 337, 1-8 (1998)

3. Virbhadra, K.S., Keeton, C.R.: Phys. Rev. D 77, 124014 (2008)

4. Virbhadra, K.S., Ellis, G.F.R.: Phys. Rev. D 65, 103004 (2002)

5. Claudel, C.-M., Virbhadra, K.S., Ellis, G.F.R.: J. Math. Phys. 42, 818-838 (2001)

6. Virbhadra, K.S.: Phys. Rev. D 79, 083004 (2009) 